\title{
STUDY OF LATERAL LOAD RESISTING SYSTEMS OF VARIABLE HEIGHTS IN ALL SOIL TYPES OF HIGH SEISMIC ZONE
}

\author{
Abhijeet Baikerikar', Kanchan Kanagali ${ }^{2}$ \\ ${ }^{1}$ Post Graduate Student in Structural Engineering, Dept of Civil Engineering, Gogte Institute of Technology Belgaum, \\ Karnataka, India \\ ${ }^{2}$ Assistant Professor, Dept of Civil Engineering, Gogte Institute of Technology, Belgaum, Karnataka, India
}

\begin{abstract}
From the ancient time we know earthquake is a disaster causing event. Recent days structures are becoming more and more slender and more susceptible to sway and hence dangerous in the earthquake. Researchers and engineers have worked out in the past to make the structures as earthquake resistant. After many practical studies it has shown that use of lateral load resisting systems in the building configuration has tremendously improved the performance of the structure in earthquake. In present research we have used square grid of $20 \mathrm{~m}$ in each direction of $5 \mathrm{~m}$ bay in each direction, software used is ETABS 9.7.0, the work has been carried out for the different cases using shear wall and bracings for the different heights, maximum height considered for the present study is $75 \mathrm{~m}$. The modeling is done to examine the effect of different cases along with different heights on seismic parameters like base shear, lateral displacements and lateral drifts. The study has been carried out for the Zone V and all types of soils as specified in IS 1893-2002.
\end{abstract}

Keywords: Bare Frame, Bracings, Shear Walls, Lateral Load Resisting Systems, Response Spectrum Method, Lateral Displacements, Drifts, Time Period, Base Shear, Seismic Zone, Soft soil

\section{INTRODUCTION}

Today's tall buildings are becoming more and more slender, leading to the possibility of more sway in comparison with earlier high-rise buildings. This has brought more challenges for the engineers to cater both gravity loads as well as lateral loads, earlier buildings were designed for the gravity loads but now because of height and seismic zone the engineers has take care of lateral loads due to earthquake and wind forces. Seismic zone plays an important role in the earthquake resistant design of building structures because the zone factor changes as the seismic intensity changes from low to very severe. Another important aspect in the design of earthquake resistant structures is soil type, as the soil type changes the whole behaviour and design of the structure changes. So to cater all the lateral forces, we have to design the structure very uniquely so that the structure can withstand for the maximum time period so that there is no harm to the society.

\section{STRUCTURAL FORM}

From the structural engineer's point of view, the determination of the structural form of a high rise building would ideally involve only the selection and arrangement of the major structural elements to resist more efficiently the various combinations of gravity and horizontal loading. The range of factors that has to be taken into account in deciding the structural form includes the internal planning, the material and method of construction, external architectural treatment, the planned location and routing of the service systems, the nature and magnitude of horizontal loading, and the height and proportions of the building. The taller and more slender a building, the more important the structural factors become, and the more necessary it is to choose an appropriate structural form. For buildings more than 10 stories, however the additional material required for wind resistance increases nonlinearly with height so that for building with 50 stories and more the selection of an appropriate structural form may be critical for the economy and indeed the viability of the building. The building structure should also posses adequate vertical and lateral stiffness to limit the deflections. Following are some general points ${ }^{[7]}$

a) The building and its superstructure should be simple, symmetric and regular in plan and elevation to prevent torsion forces.

b) The building and its superstructure should have uniform and continuous distribution of mass, stiffness, strength and ductility to avoid the overstressing of the structural components.

c) The building should be light and should avoid unnecessary masses. The larger the mass the larger are the seismic forces.

d) It is preferable not to have large height to width ratio to avoid large drift

e) The superstructure should not have long cantilevers to avoid large deformations.

\subsection{Moment Resisting Frame}

Moment resistant frame consists of columns and beams. The lateral stiffness of a moment resisting frame depends on the bending stiffness of the columns and beams. The advantage 
of moment resisting frame is that it is open rectangular arrangement which allows freedom of planning and easy fitting of doors and windows. It is economical only for buildings up to about 25 stories. Above 25 stories the relatively high flexibility of the frame calls for uneconomically large members in order to control the drift and displacements.

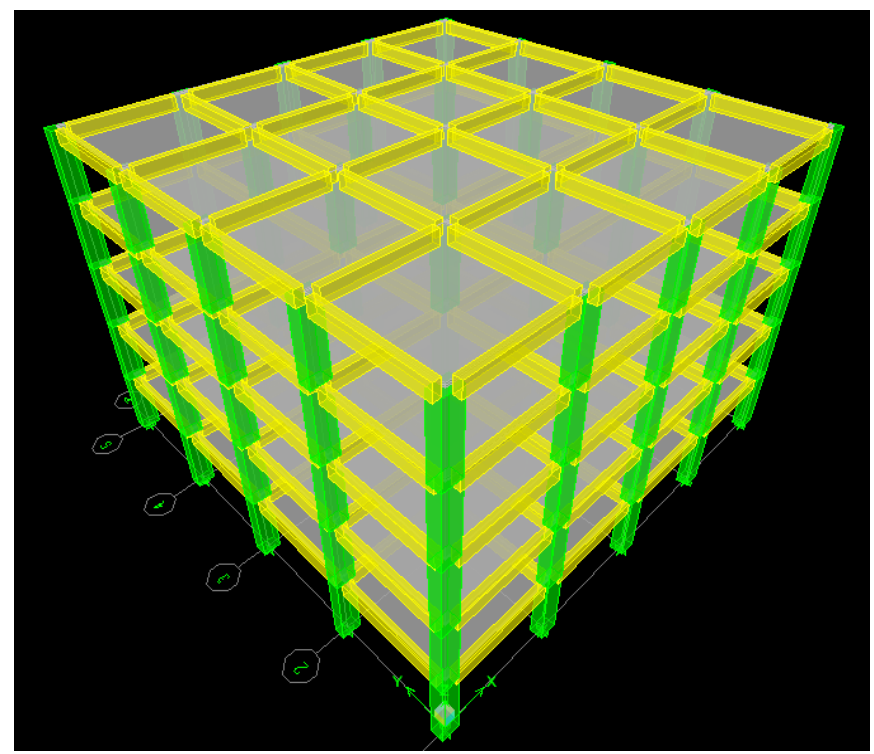

Fig -1: Moment Resisting Frame

\subsection{Shear Walls}

Continuous concrete vertical wall serve both architecturally as partitions and structurally to carry gravity and lateral loads. Their very high in plane stiffness and strength makes them ideal for tall buildings. In a shear wall structure, such walls are entirely responsible for the lateral load resistance of the building. They act as vertical cantilevers in the form of separate planar walls and as non planar assemblies of connected walls around elevator, stair and service shafts. Because they are much stiffer horizontally than rigid frames, shear wall structures can be economical up to about 35 stories. In contrast to rigid frames, the shear walls solid form tends to restrict planning where open internal spaces are required. They are well suited, however to hotels and residential buildings where the floor by floor repetitive planning allows the walls to be vertically continuous and where they serve simultaneously as excellent acoustic and fire insulators between rooms and apartments. In low to medium rise structures shear walls are combined with frames, it is reasonable to assume that shear walls attract all the lateral loading so that the frame may be designed for only gravity loading. Shear wall structures have been shown to perform well in earthquake for which ductility becomes an important consideration in their design. ${ }^{[8]}$

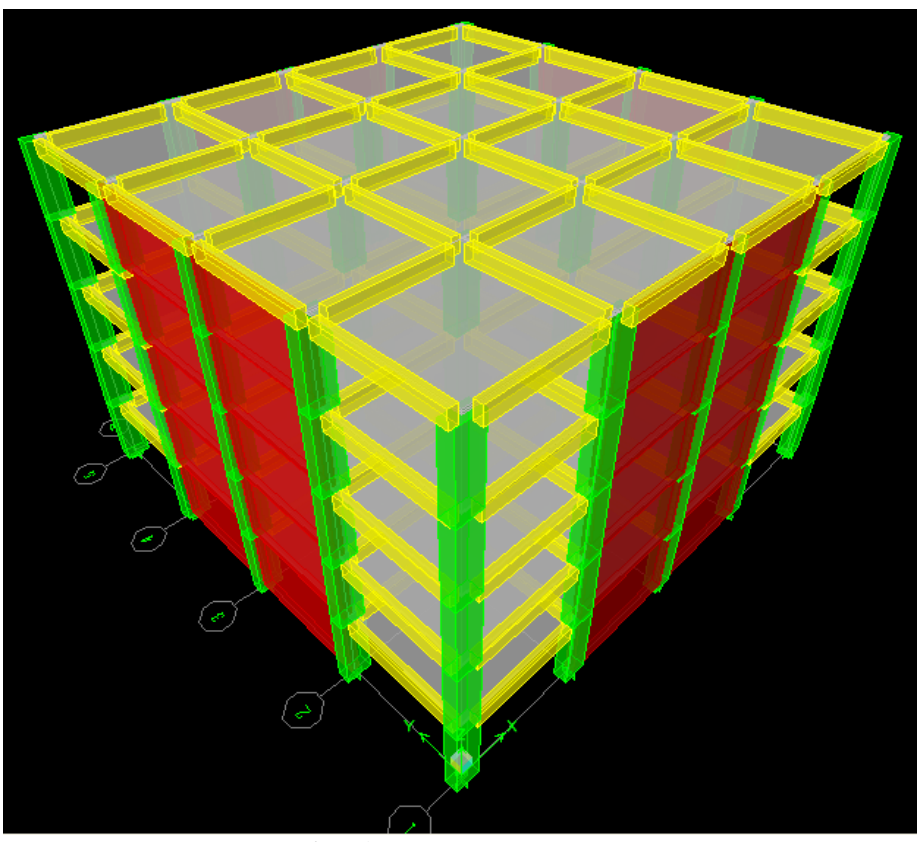

Fig -2: Shear Walls

\subsection{Bracings}

In braced frames the lateral resistance of the structure is provided by diagonal members that together with the beams form the web of the vertical truss with the columns acting as chords. Because the horizontal shear on the building is resisted by the horizontal components of the axial tensile and compressive actions in the web members bracing systems are highly efficient in resisting lateral loads. Bracing is generally regarded as an exclusive steel system but nowadays steel bracings are also used in reinforced concrete frames. The efficiency of bracing in being able to produce a laterally very stiff structure for a minimum of additional material makes it an economical structural form for any height of building, up to the very tallest. An additional advantage of fully triangulated bracing is that the beams usually participate only minimally in the lateral bracing action. A major disadvantage of diagonal bracing is that it obstructs the internal planning and the location of windows and doors. For this reason braced bents are usually incorporated internally along wall and partition lines and especially around elevator, stair, and service shafts. More recently external larger scale bracing extending over many stories and bays has been used to produce not only highly efficient structures but aesthetically attractive buildings. Braces are of two types, concentric and eccentric. Concentric braces connect at the beam column intersection, where as eccentric braces connect to the beam at some distance away from the beam column intersection. These structures with braced frames increase the lateral strength and also the stiffness of the structural system and hence reduce the drift. 


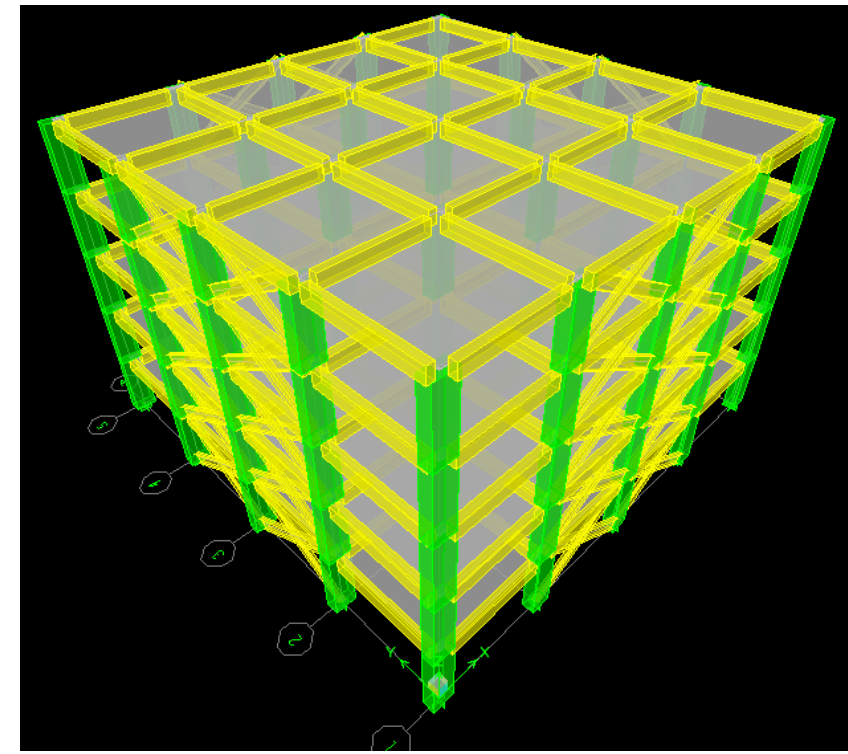

Fig -3: Bracings

\section{STUDY PARAMETERS}

a) Type of building: Multi Storied Building.

b) Zone: V

c) Type of soil: Hard, Medium and Soft soils.

d) Plan of the Building: 20mX20m.

e) Each Bay Size: $5 \mathrm{~m}$

f) Height of Buildings: $15 \mathrm{~m}, 30 \mathrm{~m} \mathrm{45m}, 60 \mathrm{~m}, 75 \mathrm{~m}$.

g) Floor to floor height: $3 \mathrm{mts}$.

h) Beams: $0.3 \mathrm{mX0.6m}$

i) Columns: $0.5 \mathrm{mX} 0.9 \mathrm{~m}$ (Storey 1 to 10$)$.

$0.5 \mathrm{mX} 0.75 \mathrm{~m}$ (Storey 11 to 20 ).

$0.5 \mathrm{mX} 0.6 \mathrm{~m}$ (Storey 21 to 25 ).

j) Slab thickness: $0.125 \mathrm{~m}$.

k) Shear Wall thickness: $0.3 \mathrm{~m}$.

1) Bracings ISMB 500.

m) Live load: $3.5 \mathrm{kN} / \mathrm{m}^{2}$.

n) Dead load of wall as UDL: $14 \mathrm{kN} / \mathrm{m}$

o) Materials: M50 and Fe415.

p) Damping 5\%.

q) Seismic analysis: Response Spectrum Method as per IS: 1893 (Part 1):2002. ${ }^{[9]}$

\section{OBJECTIVES OF STUDY}

1] Determination of Lateral Displacements, Drifts, Base Shear, Time Period at variable heights for bare frame using Response Spectrum Method in zone V for all types of soils.

2] Determination of Lateral Displacements, Drifts, Base Shear, Time Period at variable heights for frame with Shear Wall using Response Spectrum Method in zone V for all types of soils.

3] Determination of Lateral Displacements, Drifts, Base Shear, Time Period at variable heights for frame with Bracings using Response Spectrum Method in zone V for all types of soils.

4] Comparing the above three frames at variable heights.

\section{CASES OF STUDY}
1] Case 1: Bare Frame
2] Case 2: Shear Wall in Middle
3] Case3: Shear Wall at Corners
4] Case 4: Bracings in Middle
5] Case 5: Bracings at Corners

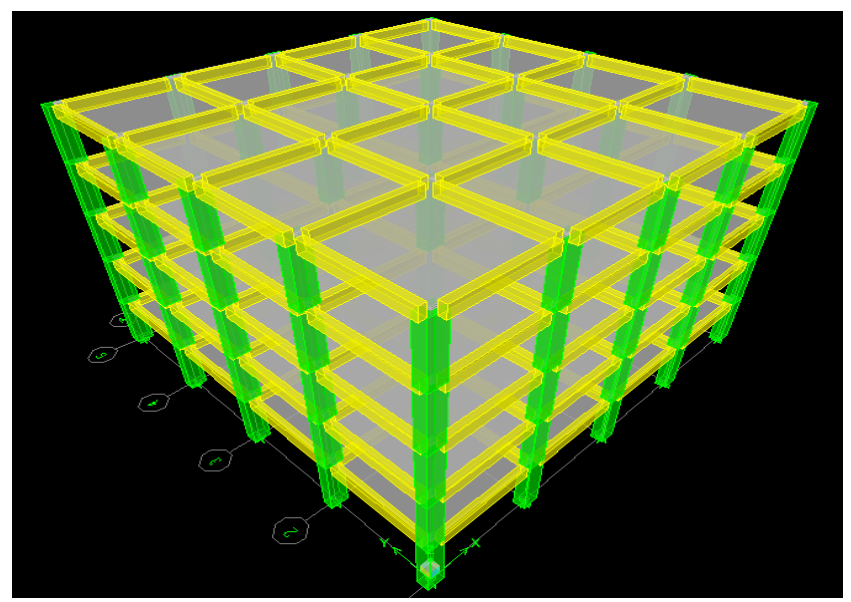

Fig -4: Case 1

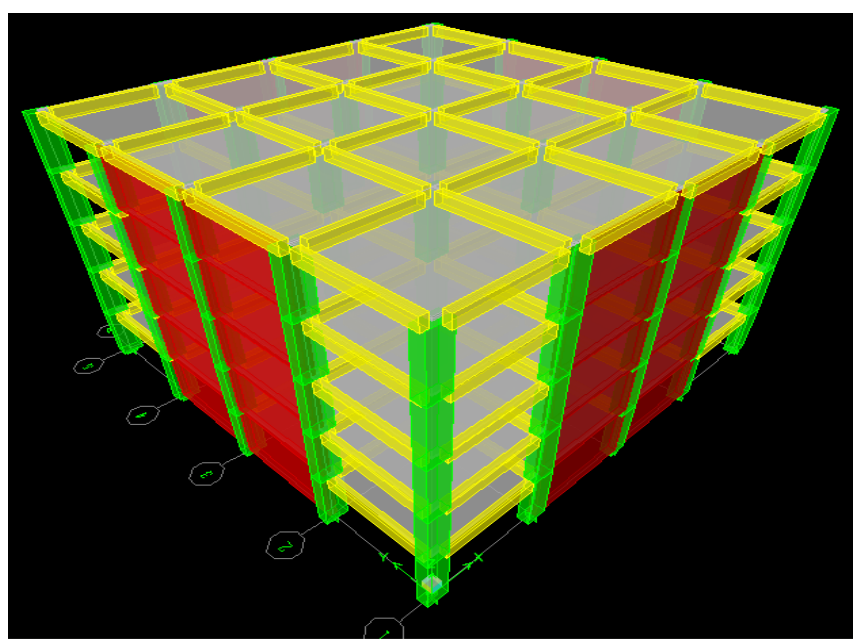

Fig -5: Case 2

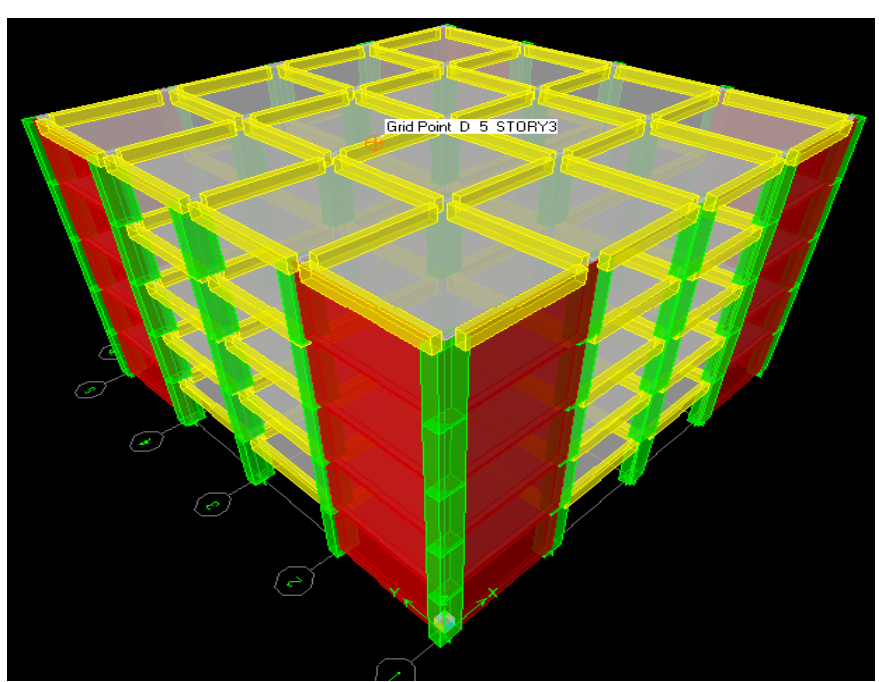

Fig -6: Case 3 


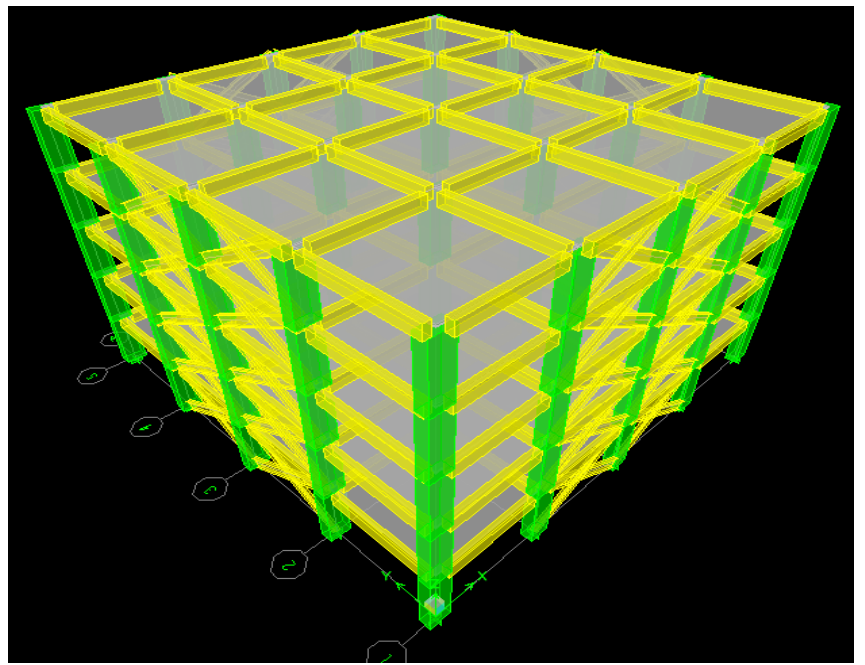

Fig -7: Case 4

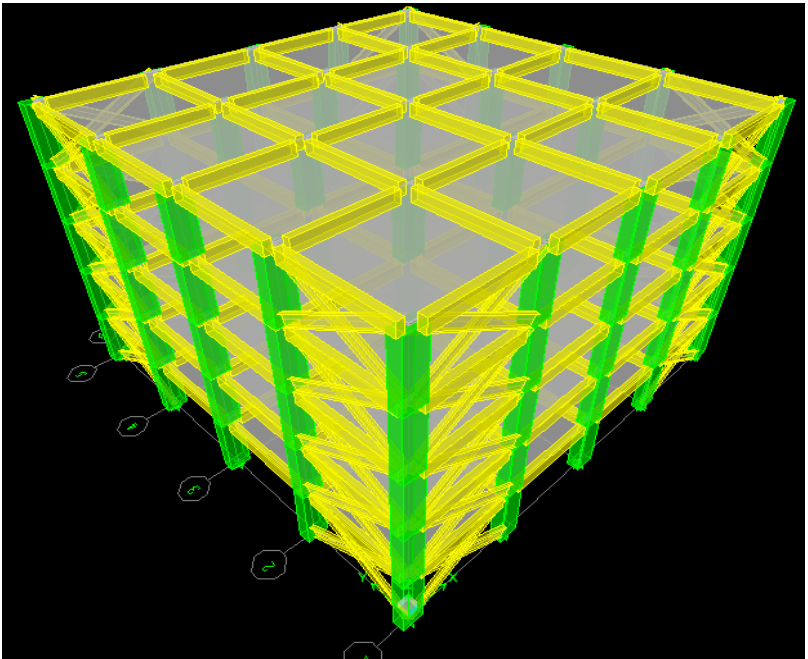

Fig -8: Case 5

\section{RESULTS}

\subsection{Zone V Hard Soil Results}

Table -1: Bare Frame Results for Hard Soil

\begin{tabular}{|c|c|c|c|c|}
\hline $\begin{array}{c}\text { HEIGHT } \\
(\mathbf{m})\end{array}$ & $\begin{array}{c}\text { BASE SHEAR } \\
(\mathbf{k N})\end{array}$ & $\begin{array}{c}\text { TIME PERIOD } \\
(\mathrm{sec})\end{array}$ & $\begin{array}{c}\text { MAX. STOREY } \\
\text { DISPLACEMENTS (mm) }\end{array}$ & $\begin{array}{c}\text { MAX. STOREY } \\
\text { DRIFT (mm) }\end{array}$ \\
\hline 15 & 1821 & $\mathbf{0 . 4 1 1 3}$ & $\mathbf{5 . 1 5}$ & $\mathbf{0 . 6 2 0}$ \\
\hline 30 & 1998 & 0.8538 & 11.10 & 0.815 \\
\hline 45 & 1975 & 1.2990 & 17.34 & 0.872 \\
\hline 60 & 1936 & 1.7703 & 23.75 & 0.885 \\
\hline 75 & 1891 & 2.2544 & 31.41 & 0.903 \\
\hline
\end{tabular}

Table -2: Shear Wall in the Middle Results for Hard Soil

\begin{tabular}{|c|c|c|c|c|}
\hline $\begin{array}{c}\text { HEIGHT } \\
(\mathbf{m})\end{array}$ & $\begin{array}{c}\text { BASE SHEAR } \\
(\mathrm{kN})\end{array}$ & $\begin{array}{c}\text { TIME PERIOD } \\
(\mathrm{sec})\end{array}$ & $\begin{array}{c}\text { MAX. STOREY } \\
\text { DISPLACEMENTS (mm) }\end{array}$ & $\begin{array}{c}\text { MAX. STOREY } \\
\text { DRIFT (mm) }\end{array}$ \\
\hline 15 & 2011 & $\mathbf{0 . 1 1 5 6}$ & $\mathbf{0 . 4 2}$ & $\mathbf{0 . 0 4 9}$ \\
\hline 30 & 3878 & 0.3249 & 3.56 & 0.221 \\
\hline 45 & 4053 & 0.5989 & $\mathbf{8 . 3 7}$ & 0.348 \\
\hline 60 & 3842 & 0.9216 & 13.31 & 0.413 \\
\hline 75 & 3787 & 1.2759 & 18.60 & 0.464 \\
\hline
\end{tabular}

Table -3: Shear Wall at the Corners Results for Hard Soil

\begin{tabular}{|c|c|c|c|c|}
\hline $\begin{array}{c}\text { HEIGHT } \\
(\mathbf{m})\end{array}$ & $\begin{array}{c}\text { BASE SHEAR } \\
(\mathbf{k N})\end{array}$ & $\begin{array}{c}\text { TIME PERIOD } \\
(\mathrm{sec})\end{array}$ & $\begin{array}{c}\text { MAX. STOREY } \\
\text { DISPLACEMENTS (mm) }\end{array}$ & $\begin{array}{c}\text { MAX. STOREY } \\
\text { DRIFT (mm) }\end{array}$ \\
\hline 15 & 1956 & $\mathbf{0 . 1 3 4 0}$ & $\mathbf{0 . 5 7}$ & $\mathbf{0 . 0 6 8}$ \\
\hline 30 & 3861 & 0.3853 & 5.02 & 0.312 \\
\hline 45 & 3574 & 0.7061 & 10.00 & 0.417 \\
\hline 60 & 3438 & 1.0728 & 15.31 & 0.498 \\
\hline 75 & 3425 & 1.4653 & 21.35 & 0.533 \\
\hline
\end{tabular}


Table -4: Bracings in the Middle Results for Hard Soil

\begin{tabular}{|c|c|c|c|c|}
\hline $\begin{array}{c}\text { HEIGHT } \\
(\mathbf{m})\end{array}$ & $\begin{array}{c}\text { BASE SHEAR } \\
(\mathbf{k N})\end{array}$ & $\begin{array}{c}\text { TIME PERIOD } \\
(\mathrm{sec})\end{array}$ & $\begin{array}{c}\text { MAX. STOREY } \\
\text { DISPLACEMENTS (mm) }\end{array}$ & $\begin{array}{c}\text { MAX. STOREY } \\
\text { DRIFT (mm) }\end{array}$ \\
\hline 15 & 1926 & $\mathbf{0 . 2 4 0 2}$ & 1.77 & $\mathbf{0 . 2 3 4}$ \\
\hline 30 & 3090 & 0.5208 & 6.94 & 0.427 \\
\hline 45 & 2791 & 0.8367 & 11.27 & 0.451 \\
\hline 60 & 2773 & 1.1953 & 16.37 & 0.493 \\
\hline 75 & 2727 & 1.5864 & 22.27 & 0.541 \\
\hline
\end{tabular}

Table -5: Bracings at the Corners Results for Hard Soil

\begin{tabular}{|c|c|c|c|c|}
\hline $\begin{array}{c}\text { HEIGHT } \\
(\mathrm{m})\end{array}$ & $\begin{array}{c}\text { BASE SHEAR } \\
(\mathrm{kN})\end{array}$ & $\begin{array}{c}\text { TIME PERIOD } \\
(\mathrm{sec})\end{array}$ & $\begin{array}{c}\text { MAX. STOREY } \\
\text { DISPLACEMENTS (mm) }\end{array}$ & $\begin{array}{c}\text { MAX. STOREY } \\
\text { DRIFT (mm) }\end{array}$ \\
\hline 15 & 1898 & 0.2528 & 2.02 & 0.250 \\
\hline 30 & 2793 & 0.5702 & 7.55 & 0.453 \\
\hline 45 & 2570 & 0.9326 & 12.75 & 0.509 \\
\hline 60 & 2565 & 1.3411 & 18.70 & 0.570 \\
\hline 75 & 2468 & 1.7806 & 25.15 & 0.617 \\
\hline
\end{tabular}

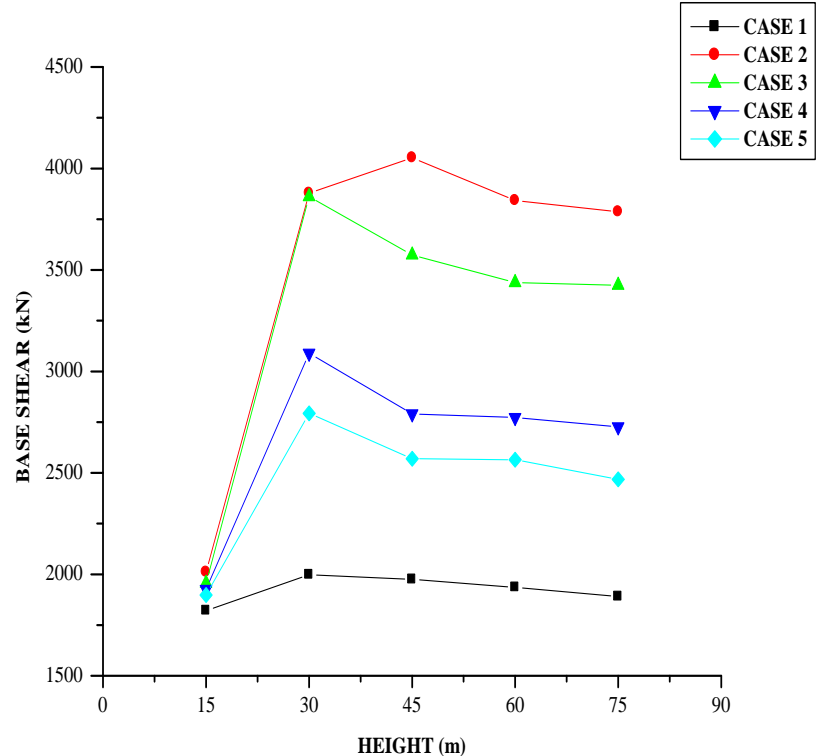

Fig -9: Height v/s Base Shear

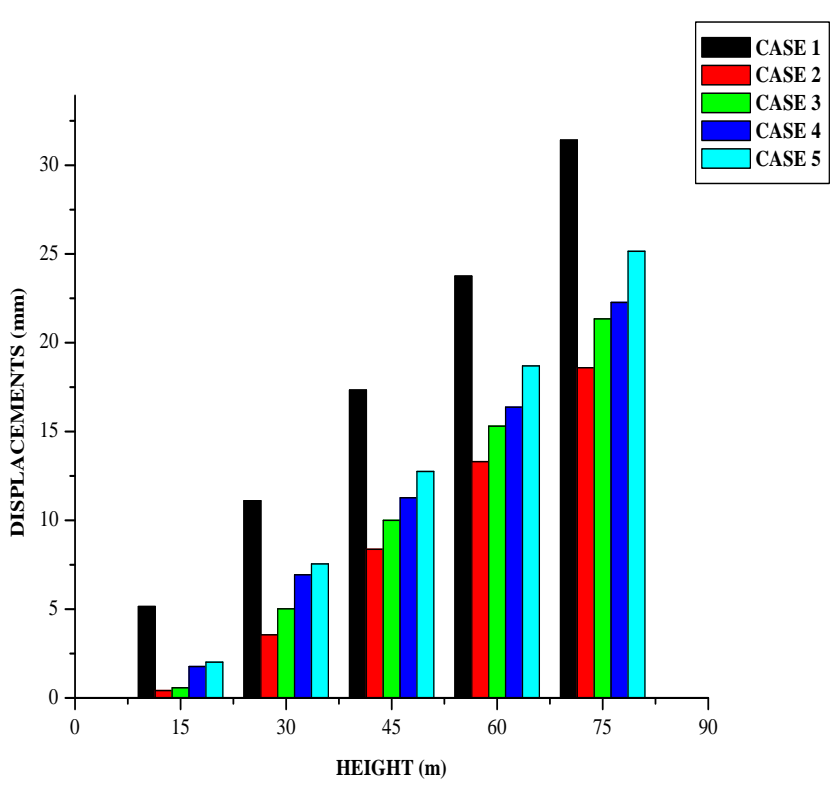

Fig -10: Displacement Graph 


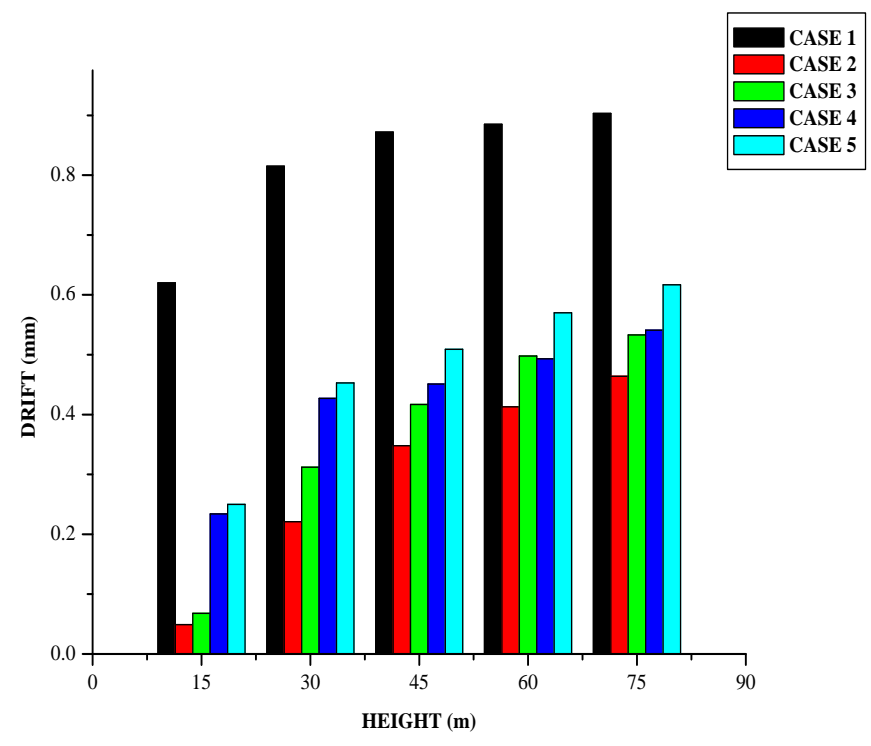

Fig -11: Drift Graph

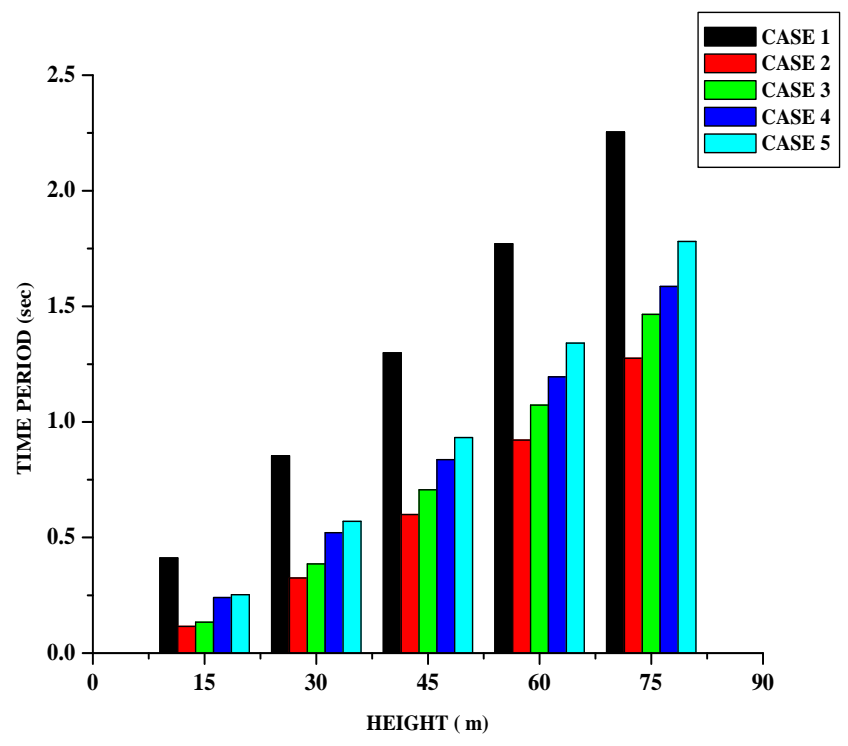

Fig -12: Time Period Graph

6.2 Zone V Medium Soil Results

Table -6: Bare Frame Results for Medium Soil

\begin{tabular}{|c|c|c|c|c|}
\hline $\begin{array}{c}\text { HEIGHT } \\
(\mathbf{m})\end{array}$ & $\begin{array}{c}\text { BASE SHEAR } \\
(\mathbf{k N})\end{array}$ & $\begin{array}{c}\text { TIME PERIOD } \\
(\mathrm{sec})\end{array}$ & $\begin{array}{c}\text { MAX. STOREY } \\
\text { DISPLACEMENTS }(\mathbf{m m})\end{array}$ & $\begin{array}{c}\text { MAX. STOREY } \\
\text { DRIFT (mm) }\end{array}$ \\
\hline 15 & 1855 & $\mathbf{0 . 4 1 1 3}$ & $\mathbf{5 . 2 9}$ & $\mathbf{0 . 6 7 3}$ \\
\hline 30 & 2697 & $\mathbf{0 . 8 5 3 8}$ & 14.79 & 1.092 \\
\hline 45 & 2587 & 1.2990 & 23.11 & 1.147 \\
\hline 60 & 2581 & 1.7703 & 32.10 & 1.189 \\
\hline 75 & 2540 & 2.2544 & 42.26 & 1.209 \\
\hline
\end{tabular}

Table -7: Shear Wall in the Middle Results for Medium Soil

\begin{tabular}{|c|c|c|c|c|}
\hline $\begin{array}{c}\text { HEIGHT } \\
(\mathbf{m})\end{array}$ & $\begin{array}{c}\text { BASE SHEAR } \\
(\mathbf{k N})\end{array}$ & $\begin{array}{c}\text { TIME PERIOD } \\
(\mathrm{sec})\end{array}$ & $\begin{array}{c}\text { MAX. STOREY } \\
\text { DISPLACEMENTS }(\mathbf{m m})\end{array}$ & $\begin{array}{c}\text { MAX. STOREY } \\
\text { DRIFT (mm) }\end{array}$ \\
\hline 15 & 2011 & $\mathbf{0 . 1 1 5 6}$ & $\mathbf{0 . 4 2}$ & $\mathbf{0 . 0 4 9}$ \\
\hline 30 & 3878 & $\mathbf{0 . 3 2 4 9}$ & $\mathbf{3 . 5 6}$ & $\mathbf{0 . 2 2 1}$ \\
\hline 45 & 5355 & $\mathbf{0 . 5 9 8 9}$ & $\mathbf{1 1 . 6 6}$ & $\mathbf{0 . 4 8 5}$ \\
\hline 60 & 4861 & $\mathbf{0 . 9 2 1 6}$ & $\mathbf{1 8 . 0 1}$ & $\mathbf{0 . 5 5 5}$ \\
\hline 75 & 4616 & 1.2759 & $\mathbf{2 4 . 9 4}$ & $\mathbf{0 . 6 1 8}$ \\
\hline
\end{tabular}

Table -8: Shear Wall at the Corners Results for Medium Soil

\begin{tabular}{|c|c|c|c|c|}
\hline $\begin{array}{c}\text { HEIGHT } \\
(\mathbf{m})\end{array}$ & $\begin{array}{c}\text { BASE SHEAR } \\
(\mathrm{kN})\end{array}$ & $\begin{array}{c}\text { TIME PERIOD } \\
(\mathrm{sec})\end{array}$ & $\begin{array}{c}\text { MAX. STOREY } \\
\text { DISPLACEMENTS (mm) }\end{array}$ & $\begin{array}{c}\text { MAX. STOREY } \\
\text { DRIFT (mm) }\end{array}$ \\
\hline 15 & 1956 & $\mathbf{0 . 1 3 4 0}$ & $\mathbf{0 . 5 7}$ & $\mathbf{0 . 0 6 8}$ \\
\hline 30 & 3861 & $\mathbf{0 . 3 8 5 3}$ & $\mathbf{5 . 0 2}$ & $\mathbf{0 . 3 1 2}$ \\
\hline 45 & 4748 & $\mathbf{0 . 7 0 6 1}$ & $\mathbf{1 3 . 8 3}$ & $\mathbf{0 . 5 7 3}$ \\
\hline 60 & 4306 & 1.0728 & $\mathbf{2 0 . 6 4}$ & $\mathbf{0 . 6 4 0}$ \\
\hline 75 & 4178 & 1.4653 & 28.37 & $\mathbf{0 . 7 0 7}$ \\
\hline
\end{tabular}


Table -9: Bracings in the Middle Results for Medium Soil

\begin{tabular}{|c|c|c|c|c|}
\hline $\begin{array}{c}\text { HEIGHT } \\
(\mathbf{m})\end{array}$ & $\begin{array}{c}\text { BASE SHEAR } \\
(\mathbf{k N})\end{array}$ & $\begin{array}{c}\text { TIME PERIOD } \\
(\mathrm{sec})\end{array}$ & $\begin{array}{c}\text { MAX. STOREY } \\
\text { DISPLACEMENTS }(\mathbf{m m})\end{array}$ & $\begin{array}{c}\text { MAX. STOREY } \\
\text { DRIFT (mm) }\end{array}$ \\
\hline 15 & 1926 & $\mathbf{0 . 2 4 0 2}$ & 1.77 & $\mathbf{0 . 2 3 4}$ \\
\hline 30 & 3710 & $\mathbf{0 . 5 2 0 8}$ & $\mathbf{8 . 6 7}$ & $\mathbf{0 . 5 3 3}$ \\
\hline 45 & 3677 & $\mathbf{0 . 8 3 6 7}$ & 15.18 & $\mathbf{0 . 6 0 8}$ \\
\hline 60 & 3529 & 1.1953 & $\mathbf{2 2 . 0 2}$ & $\mathbf{0 . 6 5 6}$ \\
\hline 75 & 3472 & 1.5864 & $\mathbf{2 9 . 6 7}$ & $\mathbf{0 . 7 0 2}$ \\
\hline
\end{tabular}

Table -10: Bracings at the Corners Results for Medium Soil

\begin{tabular}{|c|c|c|c|c|}
\hline $\begin{array}{c}\text { HEIGHT } \\
(\mathbf{m})\end{array}$ & $\begin{array}{c}\text { BASE SHEAR } \\
(\mathbf{k N})\end{array}$ & $\begin{array}{c}\text { TIME PERIOD } \\
(\mathbf{s e c})\end{array}$ & $\begin{array}{c}\text { MAX. STOREY } \\
\text { DISPLACEMENTS (mm) }\end{array}$ & $\begin{array}{c}\text { MAX. STOREY } \\
\text { DRIFT (mm) }\end{array}$ \\
\hline 15 & 1898 & $\mathbf{0 . 2 5 2 8}$ & $\mathbf{2 . 0 2}$ & $\mathbf{0 . 2 5 0}$ \\
\hline 30 & 3586 & $\mathbf{0 . 5 7 0 2}$ & 10.23 & $\mathbf{0 . 6 1 3}$ \\
\hline 45 & 3353 & $\mathbf{0 . 9 3 2 6}$ & $\mathbf{1 7 . 2 4}$ & $\mathbf{0 . 6 8 6}$ \\
\hline 60 & 3216 & 1.3411 & $\mathbf{2 4 . 9 4}$ & $\mathbf{0 . 7 5 7}$ \\
\hline 75 & $\mathbf{3 2 0 2}$ & 1.7806 & $\mathbf{3 3 . 8 5}$ & $\mathbf{0 . 8 2 8}$ \\
\hline
\end{tabular}

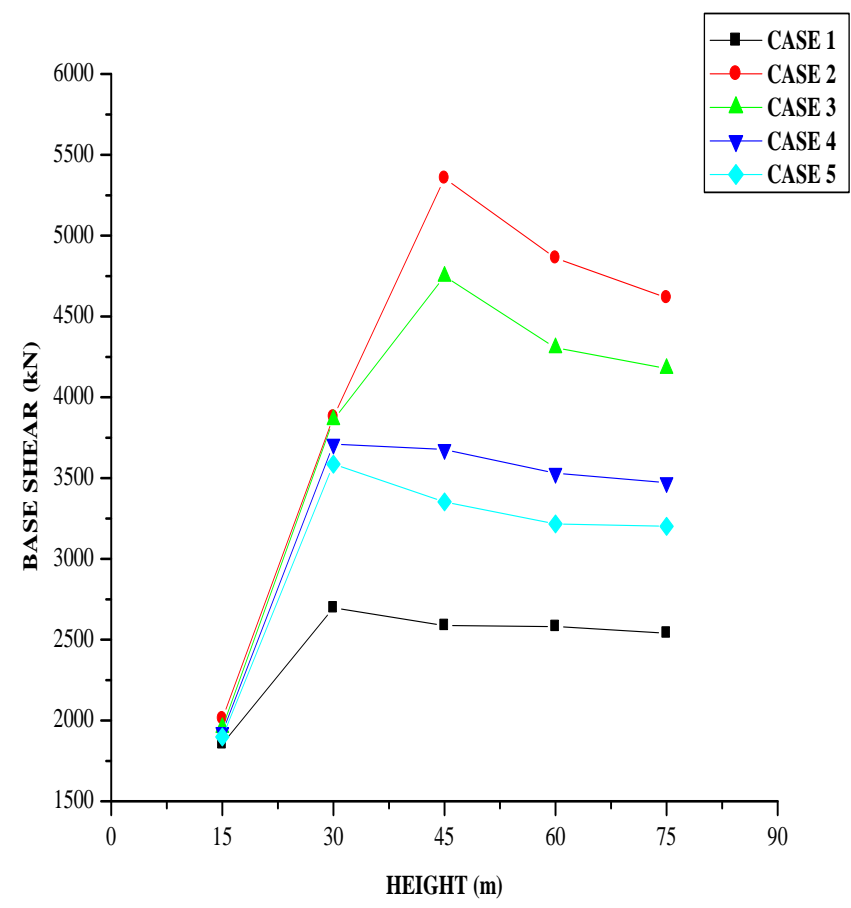

Fig -13: Height v/s Base Shear

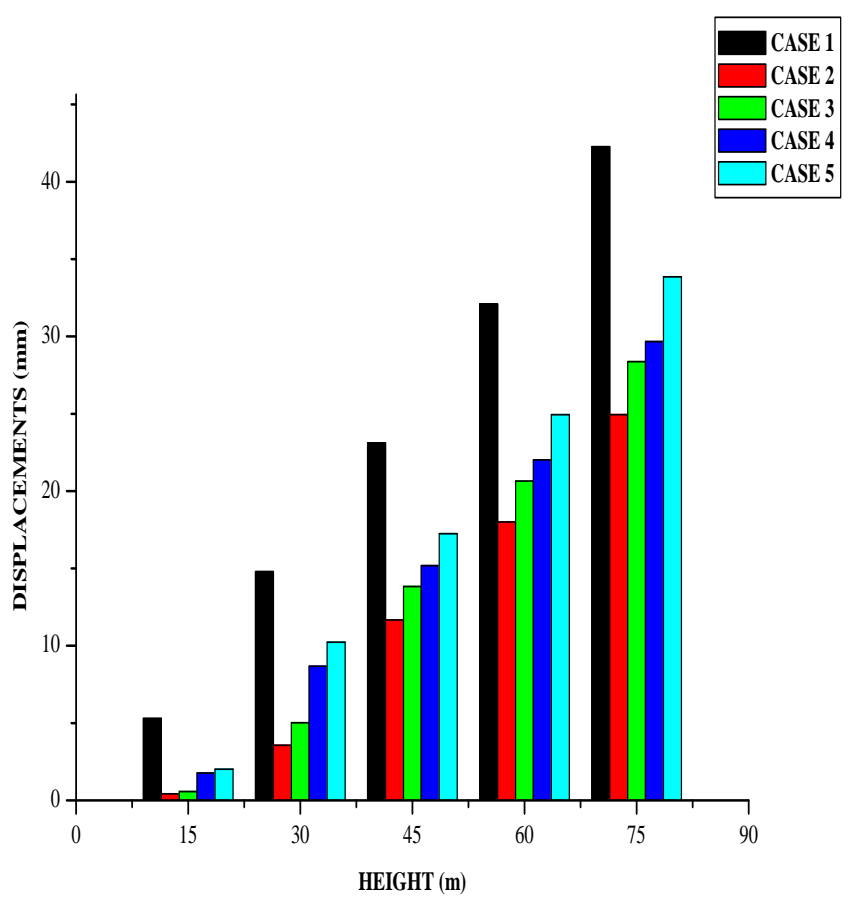

Fig -14: Displacement Graph 


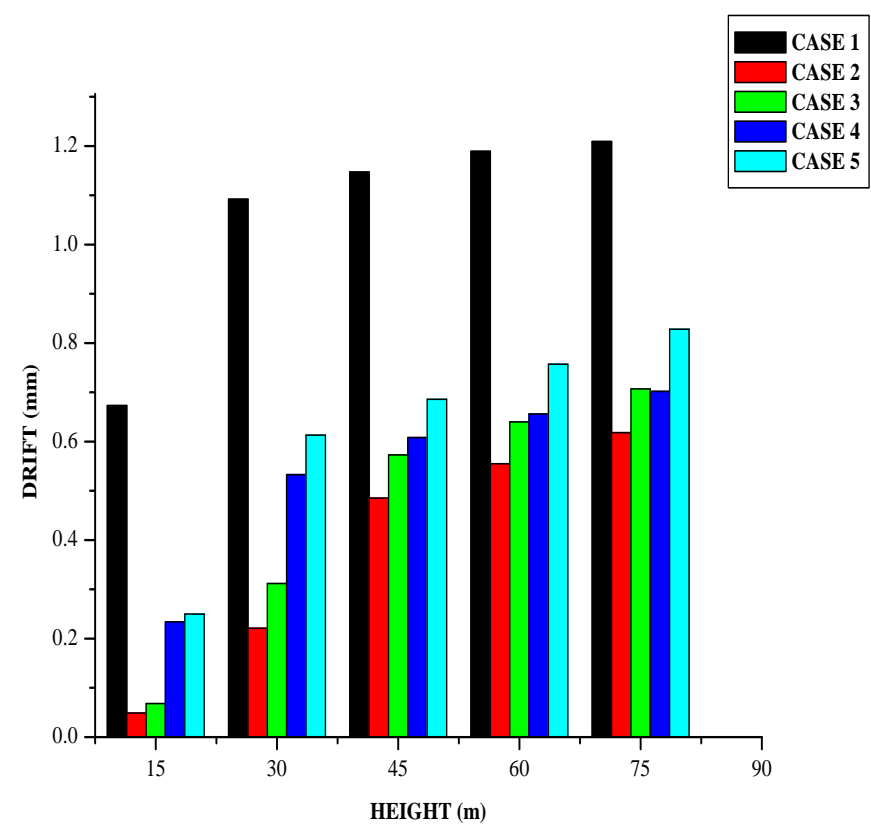

Fig -15: Drift Graph

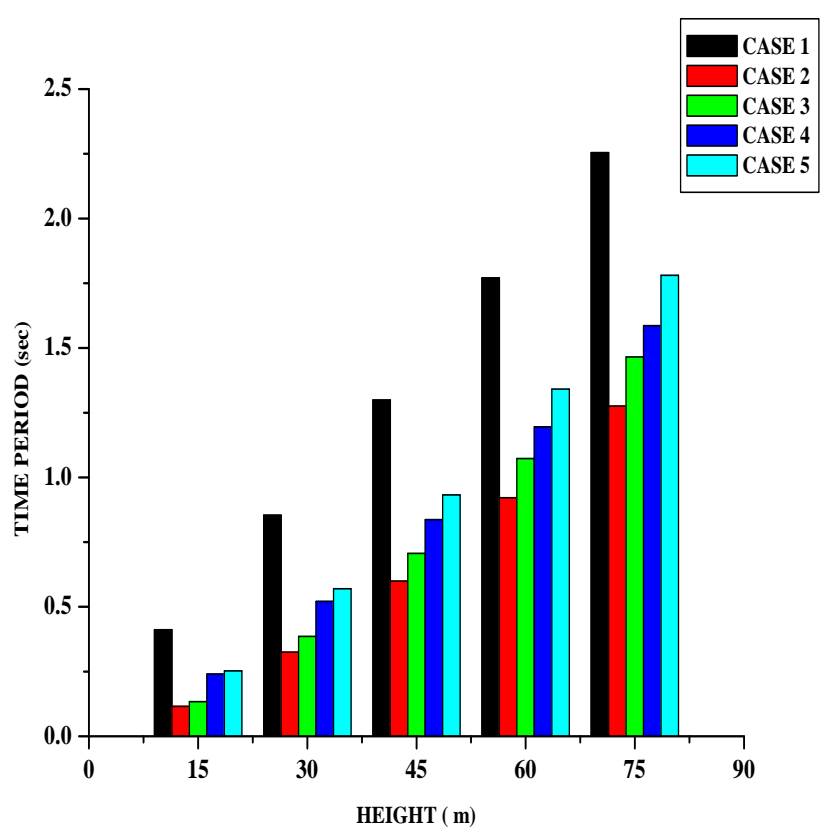

Fig -16: Time Period Graph

\subsection{Zone V Soft Soil Results}

Table -11: Bare Frame Results for Soft Soil

\begin{tabular}{|c|c|c|c|c|}
\hline $\begin{array}{c}\text { HEIGHT } \\
(\mathbf{m})\end{array}$ & $\begin{array}{c}\text { BASE SHEAR } \\
(\mathrm{kN})\end{array}$ & $\begin{array}{c}\text { TIME PERIOD } \\
(\mathrm{sec})\end{array}$ & $\begin{array}{c}\text { MAX. STOREY } \\
\text { DISPLACEMENTS (mm) }\end{array}$ & $\begin{array}{c}\text { MAX. STOREY } \\
\text { DRIFT (mm) }\end{array}$ \\
\hline 15 & $\mathbf{1 8 5 6}$ & $\mathbf{0 . 4 1 1 3}$ & $\mathbf{5 . 2 8}$ & $\mathbf{0 . 6 7 4}$ \\
\hline 30 & 3236 & $\mathbf{0 . 8 5 3 8}$ & 18.20 & $\mathbf{1 . 3 3 3}$ \\
\hline 45 & 3127 & 1.2990 & $\mathbf{2 8 . 3 0}$ & $\mathbf{1 . 3 8 4}$ \\
\hline 60 & $\mathbf{3 0 4 8}$ & $\mathbf{1 . 7 7 0 3}$ & $\mathbf{3 9 . 2 5}$ & $\mathbf{1 . 4 2 1}$ \\
\hline 75 & $\mathbf{3 0 8 1}$ & $\mathbf{2 . 2 5 4 4}$ & $\mathbf{5 1 . 2 0}$ & $\mathbf{1 . 4 6 8}$ \\
\hline
\end{tabular}

Table -12: Shear Wall in the Middle Results for Soft Soil

\begin{tabular}{|c|c|c|c|c|}
\hline $\begin{array}{c}\text { HEIGHT } \\
(\mathbf{m})\end{array}$ & $\begin{array}{c}\text { BASE SHEAR } \\
(\mathrm{kN})\end{array}$ & $\begin{array}{c}\text { TIME PERIOD } \\
(\mathrm{sec})\end{array}$ & $\begin{array}{c}\text { MAX. STOREY } \\
\text { DISPLACEMENTS (mm) }\end{array}$ & $\begin{array}{c}\text { MAX. STOREY } \\
\text { DRIFT (mm) }\end{array}$ \\
\hline 15 & 2012 & $\mathbf{0 . 1 1 5 6}$ & $\mathbf{0 . 4 3}$ & $\mathbf{0 . 0 4 8}$ \\
\hline 30 & 3879 & 0.3249 & 3.57 & 0.222 \\
\hline 45 & 5683 & 0.5989 & 12.58 & 0.518 \\
\hline 60 & 5782 & 0.9216 & 22.10 & 0.678 \\
\hline 75 & 5387 & 1.2759 & 29.93 & 0.752 \\
\hline
\end{tabular}

Table -13: Shear Wall at the Corners Results for Soft Soil

\begin{tabular}{|c|c|c|c|c|}
\hline $\begin{array}{c}\text { HEIGHT } \\
(\mathbf{m})\end{array}$ & $\begin{array}{c}\text { BASE SHEAR } \\
(\mathbf{k N})\end{array}$ & $\begin{array}{c}\text { TIME PERIOD } \\
(\mathrm{sec})\end{array}$ & $\begin{array}{c}\text { MAX. STOREY } \\
\text { DISPLACEMENTS (mm) }\end{array}$ & $\begin{array}{c}\text { MAX. STOREY } \\
\text { DRIFT (mm) }\end{array}$ \\
\hline 15 & 1957 & $\mathbf{0 . 1 3 4 0}$ & $\mathbf{0 . 5 8}$ & $\mathbf{0 . 0 7 0}$ \\
\hline 30 & 3862 & $\mathbf{0 . 3 8 5 3}$ & $\mathbf{5 . 0 3}$ & $\mathbf{0 . 3 1 0}$ \\
\hline 45 & 5554 & $\mathbf{0 . 7 0 6 1}$ & $\mathbf{1 6 . 4 9}$ & $\mathbf{0 . 6 7 5}$ \\
\hline 60 & 5096 & 1.0728 & $\mathbf{2 5 . 4 8}$ & $\mathbf{0 . 7 8 2}$ \\
\hline 75 & 4854 & 1.4653 & $\mathbf{3 4 . 6 9}$ & $\mathbf{0 . 8 6 0}$ \\
\hline
\end{tabular}


Table -14: Bracings in the Middle Results for Soft Soil

\begin{tabular}{|c|c|c|c|c|}
\hline $\begin{array}{c}\text { HEIGHT } \\
(\mathbf{m})\end{array}$ & $\begin{array}{c}\text { BASE SHEAR } \\
(\mathbf{k N})\end{array}$ & $\begin{array}{c}\text { TIME PERIOD } \\
(\mathrm{sec})\end{array}$ & $\begin{array}{c}\text { MAX. STOREY } \\
\text { DISPLACEMENTS (mm) }\end{array}$ & $\begin{array}{c}\text { MAX. STOREY } \\
\text { DRIFT (mm) }\end{array}$ \\
\hline 15 & 1927 & $\mathbf{0 . 2 4 0 2}$ & $\mathbf{1 . 7 8}$ & $\mathbf{0 . 2 3 5}$ \\
\hline 30 & $\mathbf{3 7 1 1}$ & $\mathbf{0 . 5 2 0 8}$ & $\mathbf{8 . 6 8}$ & $\mathbf{0 . 5 3 4}$ \\
\hline 45 & 4456 & $\mathbf{0 . 8 3 6 7}$ & $\mathbf{1 8 . 6 0}$ & $\mathbf{0 . 7 4 3}$ \\
\hline 60 & 4211 & 1.1953 & $\mathbf{2 6 . 9 2}$ & $\mathbf{0 . 7 9 9}$ \\
\hline 75 & 4053 & $\mathbf{1 . 5 8 6 4}$ & $\mathbf{3 6 . 3 9}$ & $\mathbf{0 . 8 7 5}$ \\
\hline
\end{tabular}

Table -15: Bracings at the Corners Results for Soft Soil

\begin{tabular}{|c|c|c|c|c|}
\hline $\begin{array}{c}\text { HEIGHT } \\
(\mathbf{m})\end{array}$ & $\begin{array}{c}\text { BASE SHEAR } \\
(\mathbf{k N})\end{array}$ & $\begin{array}{c}\text { TIME PERIOD } \\
(\mathbf{s e c})\end{array}$ & $\begin{array}{c}\text { MAX. STOREY } \\
\text { DISPLACEMENTS }(\mathbf{m m})\end{array}$ & $\begin{array}{c}\text { MAX. STOREY } \\
\text { DRIFT (mm) }\end{array}$ \\
\hline 15 & $\mathbf{1 8 9 9}$ & $\mathbf{0 . 2 5 2 8}$ & $\mathbf{2 . 0 4}$ & $\mathbf{0 . 2 5 1}$ \\
\hline 30 & $\mathbf{3 6 4 0}$ & $\mathbf{0 . 5 7 0 2}$ & $\mathbf{1 0 . 5 0}$ & $\mathbf{0 . 6 3 0}$ \\
\hline 45 & 4046 & $\mathbf{0 . 9 3 2 6}$ & $\mathbf{2 1 . 0 1}$ & $\mathbf{0 . 8 4 0}$ \\
\hline 60 & 3810 & $\mathbf{1 . 3 4 1 1}$ & $\mathbf{2 9 . 9 4}$ & $\mathbf{0 . 9 2 0}$ \\
\hline 75 & 3708 & $\mathbf{1 . 7 8 0 6}$ & $\mathbf{4 0 . 9 4}$ & $\mathbf{1 . 0 0 4}$ \\
\hline
\end{tabular}

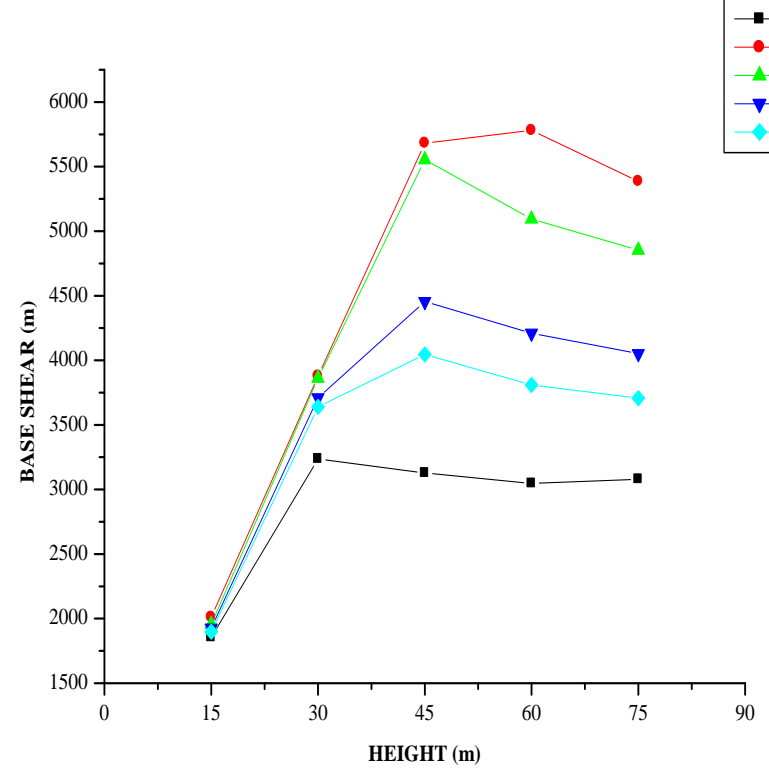

Fig -17: Height v/s Base Shear
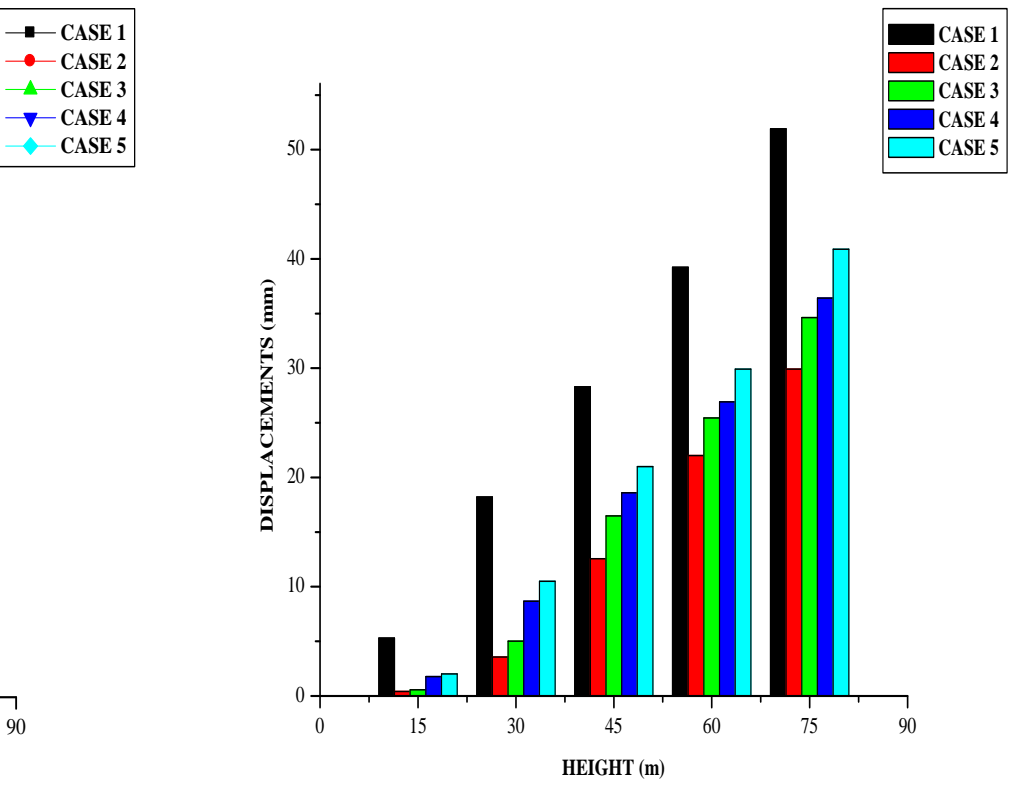

Fig -18: Displacement Graph 


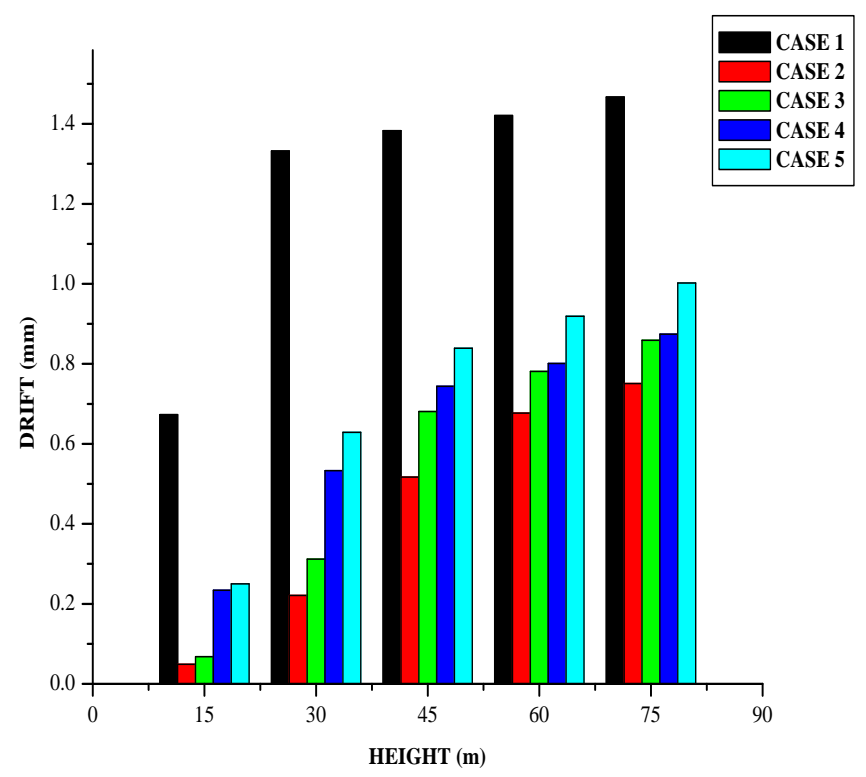

Fig -19: Drift Graph

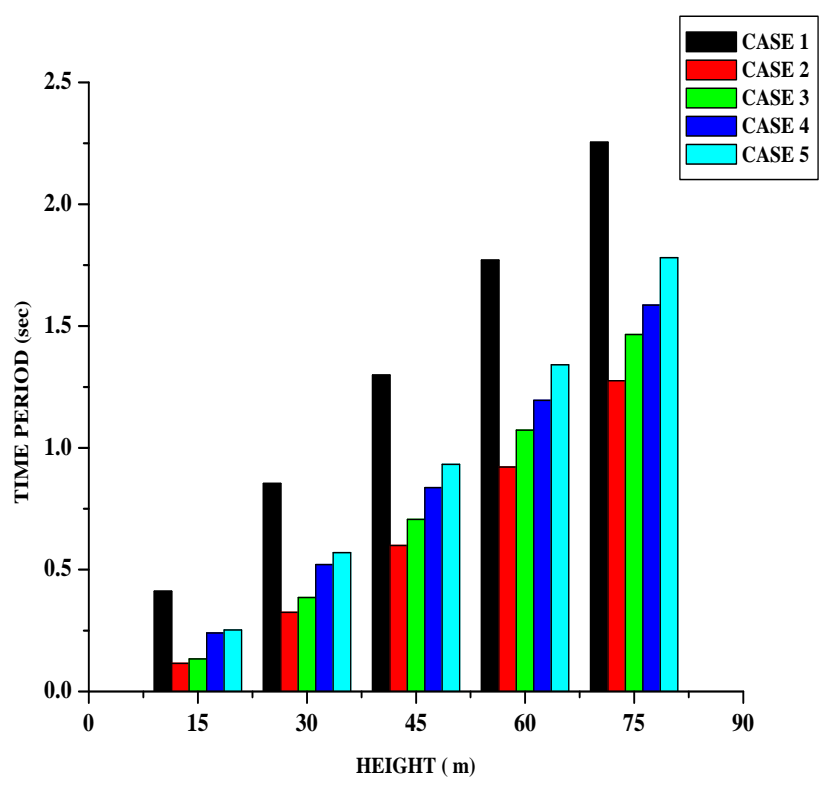

Fig -20: Time Period Graph

\section{CONCLUSIONS}

- As the building height increases Lateral displacements and drift increases.

- $\quad$ Compared to all other cases Case 1(Bare Frame) produces larger lateral displacements and drifts.

- $\quad$ Lateral displacements and drift is significantly lower after inserting shear wall and bracings in the bare frame.

- One of the important conclusions that can be made from the above study is that as the soil changes from hard to soft there is massive increase in base shear, lateral displacements and lateral drifts. Extreme care should be taken in soft soil.

- $\quad$ Time Period increases as the height of the building increases because mass of the overall building increases as time period is directly proportional to the mass.

- $\quad$ From the study it is clear that CASE 2 (Shear Wall in Middle) is performing better and more efficient than all other cases.

- $\quad$ Base Shear is decreased as the time period increases.

- $\quad$ Time period is significantly lowered after placing shear walls and bracings.

\section{SCOPE FOR FURTHUR WORK}

- $\quad$ The study can be extended for different plan size of the building.

- $\quad$ By locating shear walls at different positions and comparing the results.

- $\quad$ Further study can be done by using different types of bracings.

\section{REFERENCES}

[1] Shaik Kamal Mohammed Azam, Vinod Hosur, "Seismic Performance Evaluation of Multistoried RC Framed Buildings with Shear Wall", International Journal of Scientific \& Engineering Research, Volume 4, Issue 1, January 2013.

C.V.R Murthy, Rupen Goswami, A.R.Vijaynarayanan, Vipul V. Mehta, "Some Concepts in Earthquake Behaviour of Buildings", Gujrat State Disaster Management Authority, Government of Gujrat.

[3] Anuj Chandiwala, "Earthquake Analysis of Building Configuration with Different Position of Shear Wall", International Journal of Emerging Technology and Advanced Engineering, Volume 2, Issue12, December 2012.

[4] "Seismic Structural Solutions", CCANZ Level 6/142 Featherston St PO Box 448, Wellington 6140, New Zealand.

[5] Yogendra Singh, "Lateral Load Resisting Systems for Multi-Storey Buildings"

[6] Pankaj Agarwal and Manish Shrikhande, "Earthquake Resistant Design of Structures" (PHI Learning Private Limited, New Delhi, India).

[7] Dr. Vinod Hosur, "Earthquake Resistant Design of Building Structures" (Wiley India Pvt. Ltd, New Delhi, India).

[8] Bryan Stafford Smith, Alex Coull, "Tall Building Structures Analysis and Design" (John Wiley and Sons, Inc).

[9] Is 1893 (Part 1) 2002, "Criteria For Earthquake Resistant Design of Structures", Bureau of Indian Standards, New Delhi. 


\section{BIOGRAPHIES}

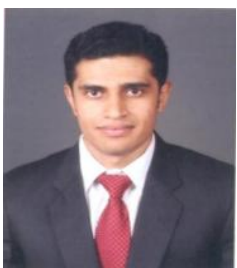

Abhijeet Baikerikar, he is currently pursuing M.Tech in Structural Engineering from Gogte Institute of Technology, Belgaum. He has completed his B.E in Civil Engineering from KLE's College of Engineering \& Technology, Belgaum. His research interest includes Seismic Analysis of Multistory Structures, Structural Dynamics, RCC \& Steel Design of Structures.

Email: abhijeet.baikerikar@yahoo.com

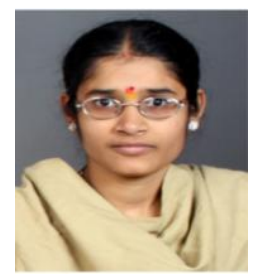

Kanchan B Kanagali, currently working as an Assistant Professor at Gogte Institute of Technology, Belgaum. Has completed her B.E in Civil Engineering and M.Tech (Structural Engg) from Gogte Institute of Technology, Belgaum. Her research interest includes Seismic Analysis of multi storey structures and Study of analysis of Progressive collapse of multi-storey structures.

Email: kkkanagali@git.edu 